\title{
The Hong Kong diastolic heart failure study: a randomised controlled trial of diuretics, irbesartan and ramipril on quality of life, exercise capacity, left ventricular global and regional function in heart failure with a normal ejection fraction
}

\author{
G W K Yip, ${ }^{1}$ M Wang, ${ }^{1}$ T Wang, ${ }^{1}$ S Chan, ${ }^{1}$ J W H Fung, ${ }^{1}$ L Yeung, ${ }^{1}$ T Yip, ${ }^{2}$ S-T Lau, ${ }^{3}$ \\ C-P Lau, ${ }^{4}$ M-O Tang, ${ }^{4}$ C-M Yu, ${ }^{1}$ J E Sanderson ${ }^{1}$
}

\begin{abstract}
${ }^{1}$ Division of Cardiology, Department of Medicine $\&$ Therapeutics, The Chinese University of Hong Kong, Prince of Wales Hospital, Hong Kong SAR; ${ }^{2}$ Department of Medicine, Yan Chai Hospital, Hong Kong SAR; ${ }^{3}$ Department of Medicine, Princess Margaret Hospital, Hong Kong SAR; ${ }^{4}$ Division of Cardiology, University of Hong Kong, Queen Mary Hospital, Hong Kong SAR
\end{abstract}

Correspondence to: Professor John Sanderson, Department of Cardiovascular Medicine, The Medical School, University of Birmingham, Edgbaston, Birmingham B15

2TT; j.e.sanderson@bham.ac.uk

Accepted 18 December 2007 Published Online First

20 January 2008

\begin{abstract}
Background: Although heart failure with a preserved or normal ejection fraction (HFNEF or diastolic heart failure) is common, treatment outcomes on quality of life and cardiac function are lacking. The effect of reninangiotensin blockade by irbesartan or ramipril in combination with diuretics on quality of life $(\mathrm{OoL})$, regional and global systolic and diastolic function was assessed in HFNEF patients.
\end{abstract}

Methods: 150 patients with HFNEF (LVEF $>45 \%$ ) were randomised to (1) diuretics alone, (2) diuretics plus irbesartan, or (3) diuretics plus ramipril. 0oL, 6-minute walk test (6MWT) and Doppler echocardiography were performed at baseline, 12, 24 and 52 weeks.

Results: The OoL score improved similarly in all three groups by 52 weeks ( $-46 \%, 51 \%$, and $50 \%$ respectively, all $p<0.01)$, although 6MWT increased only slightly (average $+3-6 \%$ ). Recurrent hospitalisation rates were equal in all groups (10-12\% in 1 year). At 1 year, LV dimensions or LVEF had not changed in any group, though both systolic and diastolic blood pressures were lowered in all three groups from 4 weeks onwards. At baseline both mean peak systolic (Sm) and early diastolic (Em) mitral annulus velocities were reduced, and increased slightly in the diuretic plus irbesartan (Sm 4.5 (SEM 0.17) to 4.9 (SEM 0.16) cm/sec; Em 3.8 (SEM 0.25) to 4.2 (SEM 0.25) cm/sec) and ramipril (Sm 4.5 (SEM 0.24) to 4.9 (SEM 0.20) cm/sec; Em 3.3 (SEM 0.25) to 4.04 (SEM $0.32) \mathrm{cm} / \mathrm{sec}$ ) groups (both $p<0.05$ ). NT-pro-BNP levels were raised at baseline (595 (SD 905) pg/ml; range 54748) and fell in the irbesartan (-124 (SD 302) pg/ml, $\mathrm{p}=0.01)$ and ramipril $(-173$ (SD 415) pg/ml, $\mathrm{p}=0.03$ ) groups only.

Conclusions: In this typically elderly group of HF patients with normal LVEF, diuretic therapy significantly improved symptoms and neither irbesartan nor ramipril had a significant additional effect. However, diuretics in combination with irbesartan or ramipril marginally improved LV systolic and diastolic longitudinal LV function, and lowered NT-proBNP over 1 year.

There are few trials of treatment in the group of patients with heart failure and a normal left ventricular ejection fraction (LVEF), variously labelled "diastolic heart failure" (DHF), "heart failure with preserved systolic function" or simply "heart failure with normal ejection fraction" (HFNEF). ${ }^{12}$ Two large-scale trials ${ }^{3}{ }^{4}$ have recently reported results: in the CHARM-Preserved trial the angiotensin receptor blocker (ARB) candesartan produced a modest reduction in hospitalisations for heart failure but no significant effect on mortality; ${ }^{3}$ in PEP-CHF the angiotensin converting enzyme inhibitor (ACEI) perindopril had similar effects. ${ }^{4}$ However, since these patients are usually elderly, often with other comorbidities, quality of life and exercise capacity may be more relevant end points than mortality. Although the pathophysiology and mechanism of symptoms in HFNEF are not entirely clear and it appears to be a heterogeneous condition, a number of studies have now shown that LV longitudinal function is reduced not only in diastole, in keeping with other abnormalities of diastolic function, but also in systole even though LV ejection fraction is within normal limits. ${ }^{5-11}$ Ventricular long axis function is particularly sensitive to the effects of hypertension, ischaemia, diabetes and ageing, which are the common precursors of HFNEF. ${ }^{341213}$ Furthermore, recent studies have shown that atrio-ventricular plane movement or longitudinal function is a primary contributor to LV pumping, accounting for $60 \%$ of the stroke volume in normal subjects and in patients with dilated cardiomyopathy. ${ }^{14}$ Therefore we have assessed in HFNEF patients the effect of blockade of the renin-angiotensin system by an angiotensin receptor blocker or an angiotensin converting enzyme inhibitor not only on symptoms and quality of life but also on LV global and regional (longitudinal) function.

\section{METHODS}

\section{Study design and subjects}

This trial was a prospective, multi-centred, randomised, open-label with blinded end point design (PROBE) to test the hypothesis that addition of ramipril or irbesartan to standard therapy with diuretics would be superior to diuretics alone in its effect on quality of life and ventricular function in patients with HFNEF. Patients admitted into hospital with a clinical diagnosis of heart failure were screened. The inclusion criteria were age $>18$ years, clinical history of heart failure within 2 months prior to screening including a chest $x$ ray demonstrating pulmonary congestion, NYHA functional class II - IV, left ventricular ejection fraction $>45 \%$ by $2 \mathrm{D}$-echocardiography or a 
radionuclide technique, and therapy with diuretics with stable dose $>14$ days prior to recruitment. Patients were randomly allocated using computer-generated random numbers in blocks of 10 (balanced stratification) to one of three treatments: (1) continue with diuretics alone (either frusemide or thiazide depending on the degree of fluid retention), (2) diuretics plus irbesartan, (3) diuretics plus ramipril. The initial dose of irbesartan was $18.75 \mathrm{mg}$ daily which was titrated at 4 and 8 weeks to $75 \mathrm{mg}$ daily. Ramipril was started at $2.5 \mathrm{mg}$ daily and similarly titrated to $10 \mathrm{mg}$ daily. Exclusion criteria were: NYHA functional class I, myocardial infarction within 3 months, unstable angina within 1 month, significant valvular heart disease, uncontrolled hypertension, serious cardiac arrhythmias, concurrent therapy with calcium channel antagonist, $\beta$-blockers ( $\alpha$-methyl dopa was used for treating hypertension if required), positive inotropic agents (except digoxin for control of atrial fibrillation) and other angiotensin converting enzyme inhibitors or receptor blockers. At baseline full Doppler echocardiographic studies, electrocardiogram, chest $x$ ray, 6minute Hall walk test (6MWT), QoL questionnaire, and routine blood testing were carried out. These were repeated at 12 and 24 weeks, and all except the exercise test at 52 weeks after randomisation.

The primary end points were (i) symptoms and quality of life and (ii) Doppler echocardiographic measurement of ventricular function. All outcomes were reviewed blind to treatment allocation.

The study was conducted in accordance with the Declaration of Helsinki and was approved by the local clinical research ethics committee. All patients gave informed consent.

\section{Quality of life}

QoL was assessed using the Minnesota Heart Failure Symptom Questionnaire, which has been previously validated in this population..$^{15} 16$

\section{Exercise testing}

Exercise capacity was measured using the 6MWT with two baseline tests as recommended. ${ }^{17}$

\section{Echocardiography}

Echocardiograms were obtained using GE-VingMed System FiVe or 7 with a $3.5 \mathrm{MHz}$ transducer. Methods of acquiring 2-D Doppler, TDI and their measurements were as previously described. ${ }^{18}$ LVEF was measured by Simpson's method as recommended. ${ }^{18}$ All analyses of digitally stored recordings were performed by one investigator (MW), who was "blinded" to treatment. Mitral inflow velocities were recorded in the usual manner to derive peak early diastolic (E) velocity and peak atrial filling velocity (A). The LV mass was calculated using the modified ASE cube formula proposed by Devereux et al. ${ }^{19}$ Colour tissue Doppler derived myocardial velocities measured at basal septal, lateral, inferior and anterior positions around the mitral annulus were recorded, analysed off-line and averaged. Peak velocities during systole ( $\mathrm{Sm}$ ), early diastole (Em) and late diastole (Am) were measured and the ratio of E/Em was used as an index of LV filling pressure as previously described. ${ }^{20}$ Measurements are a mean of three beats and for patients in atrial fibrillation six beats were recorded and averaged.

\section{N-terminal pro-brain natriuretic peptide (NT-proBNP)}

Blood samples were collected for measurement of NT-pro-BNP on study enrolment and at 12 and 52 weeks. Serum NT-pro-BNP was quantified by electrochemiluminescence immunoassay on the Roche Elecsys 2010 analyser (Roche Diagnostics Corporation, Indianapolis, IN, USA) with an interassay coefficient of variation (CV) of $2.6 \%$ at $1068 \mathrm{pg} / \mathrm{ml}$ and a measuring range from 5 to $35000 \mathrm{pg} / \mathrm{ml}$.

\section{STATISTICS}

With $n=50$ in each group the study had a $>90 \%$ power to detect a $50 \%$ reduction of symptom score, a $20 \%$ improvement in exercise time within each group, a $50 \%$ reduction in hospital admissions, and a $20 \%$ improvement in peak systolic and early diastolic basal myocardial velocities at a 5\% significance level, all of which would be considered to be clinically significant.

The statistical program SPSS version 11.0 (Chicago, IL, USA) was used for all the analyses. Comparisons of the baseline characteristics among the three groups were performed by analysis of variance with repeated measures and with the $\chi^{2}$ test for categorical variables. Comparisons between baseline and follow-up at different time points were by paired t test with Bonferroni adjustment. Differences between groups at baseline, 12 or 52 weeks were tested by using the 1-way ANOVA and the Kruskal-Wallis method for non-parametric data.

The results were expressed as mean (SE) (or mean (SD) where indicated) and their differences considered significant if $\mathrm{p}<0.05$.

\section{RESULTS}

Baseline clinical characteristics are shown in table 1. There were no significant differences between the three treatment groups. The majority of the patients had hypertension (82\%) as the main aetiology. Twenty per cent had a clinical diagnosis of diabetes. Most (70\%) were in NYHA class II. In the diuretic group three patients died due to cerebrovascular haemorrhage, cancer of the liver and cancer of the lung. In the diuretic group one patient was withdrawn from the study at 4 weeks because of uncontrolled high blood pressure, one defaulted and one refused to continue. In the irbesartan group one patient died due to heart failure and one was withdrawn due to onset of fast atrial fibrillation. In the ramipril group four patients were withdrawn because of persistent irritating cough and one because of uncontrolled blood pressure, and one patient refused to continue. No patient died.

\section{QoL and exercise capacity}

The changes in QoL units are shown in table 2 and fig 1 . There was a significant fall (improvement) in all three groups, which was apparent by 12 weeks, and there was no difference between groups. Distance walked in the 6MWT increased slightly in all groups but this was not statistically significant. Readmission rates were low (11-12\%) in all groups and there was only one death due to heart failure during the 1-year follow-up. The mean dose of diuretics did not change significantly from baseline to 1 year in any group (table 2).

\section{Doppler echocardiography}

In table 3 baseline echocardiographic variables for the whole HFNEF group are compared with age-matched normal subjects. Peak Sm and Em were significantly lower and LV mass and LA size increased compared with normal subjects. Filling pressure estimated by $\mathrm{E} / \mathrm{Em}$ ratio was also increased in patients. The impact of treatment on Doppler echocardiography variables is shown in table 4 . There was an increase (improvement) in the peak early diastolic myocardial velocity (Em) in all groups but this was more marked and statistically significant only for those 
Table 1 Baseline clinical characteristics of patients

\begin{tabular}{|c|c|c|c|c|}
\hline & $\begin{array}{l}\text { Diuretic only } \\
\mathbf{n}=\mathbf{5 0}\end{array}$ & $\begin{array}{l}\text { Diuretic + Irbesartan } \\
\mathbf{n}=\mathbf{5 6}\end{array}$ & $\begin{array}{l}\text { Diuretic + Ramipril } \\
\mathrm{n}=\mathbf{4 5}\end{array}$ & p Value \\
\hline \multicolumn{5}{|l|}{ Patients' characteristics } \\
\hline Mean age (SD) & $73(8.4)$ & $75(8.5)$ & $74(6.1)$ & NS \\
\hline Female/male (\%) & $29(58) / 21(42)$ & $37(66) / 19(34)$ & $27(60) / 18(40)$ & NS \\
\hline Body mass index (SD) $\left(\mathrm{kg} / \mathrm{m}^{2}\right)$ & $26.8(4.2)$ & $27.2(4.1)$ & $26.8(3.9)$ & NS \\
\hline \multicolumn{5}{|l|}{ Medical history } \\
\hline Hypertension (\%) & $38(76)$ & $40(71)$ & $33(73)$ & NS \\
\hline Angina/Myocardial infarction (\%) & $9(18)$ & $6(11)$ & $8(18)$ & NS \\
\hline Atrial fibrillation $(\%)$ & $5(10)$ & $12(21)$ & $7(16)$ & NS \\
\hline Paroxysmal atrial fibrillation (\%) & $3(6)$ & $6(11)$ & $4(9)$ & NS \\
\hline Diabetes mellitus (\%) & $10(20)$ & $10(18)$ & $10(22)$ & NS \\
\hline Hyperlipidaemia (\%) & $4(8)$ & $7(13)$ & $3(7)$ & NS \\
\hline Smoker (\%) & $5(10)$ & $7(13)$ & $4(9)$ & NS \\
\hline Ex-smoker (\%) & $19(38)$ & $13(23)$ & $16(36)$ & NS \\
\hline \multicolumn{5}{|l|}{ Aetiology of heart failure } \\
\hline Ischaemic heart failure (\%) & $6(12)$ & $4(7)$ & $7(16)$ & NS \\
\hline Hypertension (\%) & $40(80)$ & $47(84)$ & $37(82)$ & NS \\
\hline Idiopathic & $4(8)$ & $5(9)$ & $1(2)$ & NS \\
\hline \multicolumn{5}{|l|}{ Signs and symptoms of $\mathrm{CHF}$} \\
\hline \multicolumn{5}{|l|}{ NYHA class } \\
\hline II & $36(72.0)$ & $38(67.9)$ & $30(66.7)$ & NS \\
\hline III & $14(28.0)$ & $17(30.4)$ & $15(33.3)$ & NS \\
\hline IV & 0 & 0 & 0 & \\
\hline SBP (mean (SD)) & $145(23)$ & 145 (19) & $143(22)$ & NS \\
\hline DBP (mean (SD)) & $80(14)$ & $82(10)$ & $79(12)$ & NS \\
\hline Pulse & $76(14)$ & 77 (9) & $79(13)$ & NS \\
\hline$J V P \geqslant 6 \mathrm{~cm}(\%)$ & $4(8.0)$ & 0 & $1(2.2)$ & NS \\
\hline Oedema (\%) & $14(28.0)$ & $9(16.1)$ & $7(15.9)$ & NS \\
\hline Third heart sound (\%) & $3(6.0)$ & $1(1.8)$ & $4(8.9)$ & NS \\
\hline \multicolumn{5}{|l|}{ Chest $x$ ray (on admission) } \\
\hline Upper lobe vein prominent (\%) & $41(83.7)$ & $48(88.9)$ & $38(88.4)$ & NS \\
\hline Kerley lines (\%) & $9(18.4)$ & $11(20.4)$ & $5(11.6)$ & NS \\
\hline Interstitial oedema (\%) & $21(42.9)$ & $16(29.6)$ & $12(27.9)$ & NS \\
\hline \multicolumn{5}{|l|}{ Medical treatment } \\
\hline Frusemide (\%) & $34(68.0)$ & $45(80.4)$ & $36(80.0)$ & NS \\
\hline mean dosage (SD) (mg) & $44.0(19)$ & $33.3(14)$ & $33.3(15)$ & NS \\
\hline Hydrochlorothiazide (\%) & $3(6.0)$ & $6(10.7)$ & $4(8.9)$ & NS \\
\hline mean dosage (SD) (mg) & $25(0)$ & $29(10)$ & $38(14)$ & NS \\
\hline Indapamide (\%) & $3(6.0)$ & $3(5.4)$ & $2(4.4)$ & NS \\
\hline mean dosage (SD) (mg) & $1.8(0.6)$ & $2.5(0)$ & $2.0(0.7)$ & NS \\
\hline Dyazide (\%) & $6(12.0)$ & $1(1.8)$ & $1(2.2)$ & NS \\
\hline$\alpha$-methyldopa (\%) & $9(18.8)$ & $7(13.0)$ & $10(23.3)$ & NS \\
\hline mean dosage (SD) (mg) & $151(348)$ & $93(258)$ & $157(327)$ & NS \\
\hline
\end{tabular}

D, diuretic; DBP, diastolic blood pressure; NS, not significant; SD, standard deviation; SBP, systolic blood pressure.
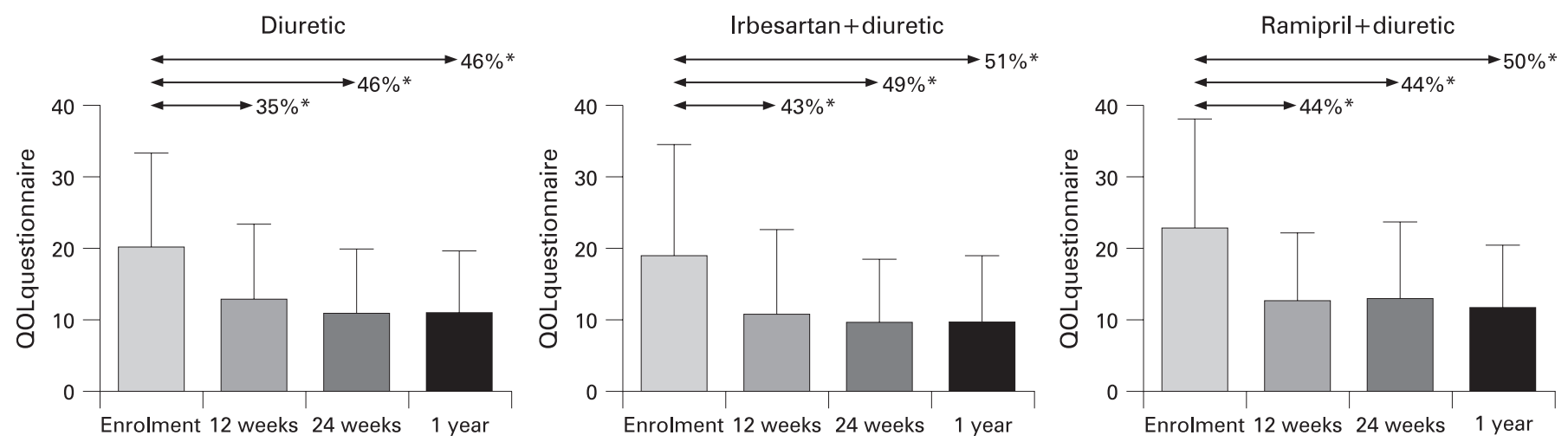

Figure 1 Quality of life scores $(\mathrm{OoL})$ in the three treatment groups over 1 year. ${ }^{*} \mathrm{p}<0.01$, significant differences between baseline and follow-up at 12 weeks, 24 weeks or 1 year. 
Table 2 Main clinical outcomes

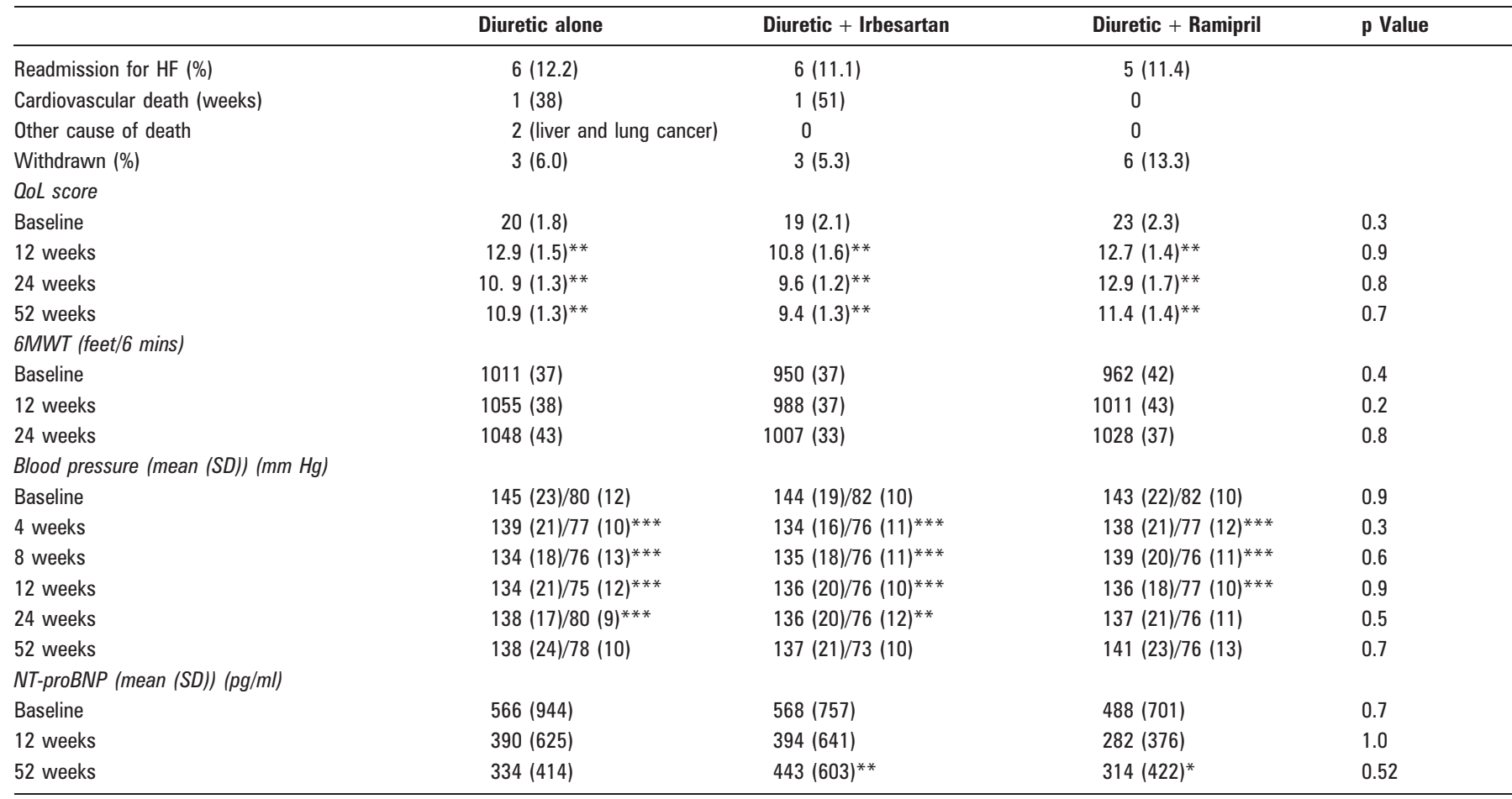

Results expressed as mean (SEM) unless otherwise stated.

${ }^{*} \mathrm{p}<0.05 ;{ }^{* *} \mathrm{p}<0.01 ;{ }^{* *} \mathrm{p}<0.001$ vs baseline.

NT-proBNP, N-terminal pro-brain natriuretic peptide; QoL, quality of life score; 6MWT, 6-minute corridor walk test.

receiving irbesartan or ramipril (fig 2). E/Em tended to fall more in the diuretic plus ramipril group. There was a significant fall in LV mass in the group receiving irbesartan (fig 2). However, after one year, there were no statistically significant differences in the percentile changes in Em, E/Em and LVmass among three groups (table 5). There were no significant changes in LV, LA dimensions, or LV ejection fraction, although peak systolic myocardial velocity increased in all groups.

\section{Reproducibility}

The intraclass correlations for various mitral annular variables by the same observer were between 0.8 and 0.9 . The interobserver correlations for the same variables were between 0.7 and 0.9. With the Bland-Altman method, the mean difference between observations was less than $5 \%$ of the mean value of the observations for measurements of velocity.
Figure 2 Effect of treatment on peak early diastolic mitral annular velocities (Em), peak systolic velocity (Sm), E/Em (an index of LV filling pressures) and LV mass in the three treatment groups. ${ }^{*} \mathrm{p}<0.05$, significant differences between baseline and follow-up at 12,24 or 52 weeks.

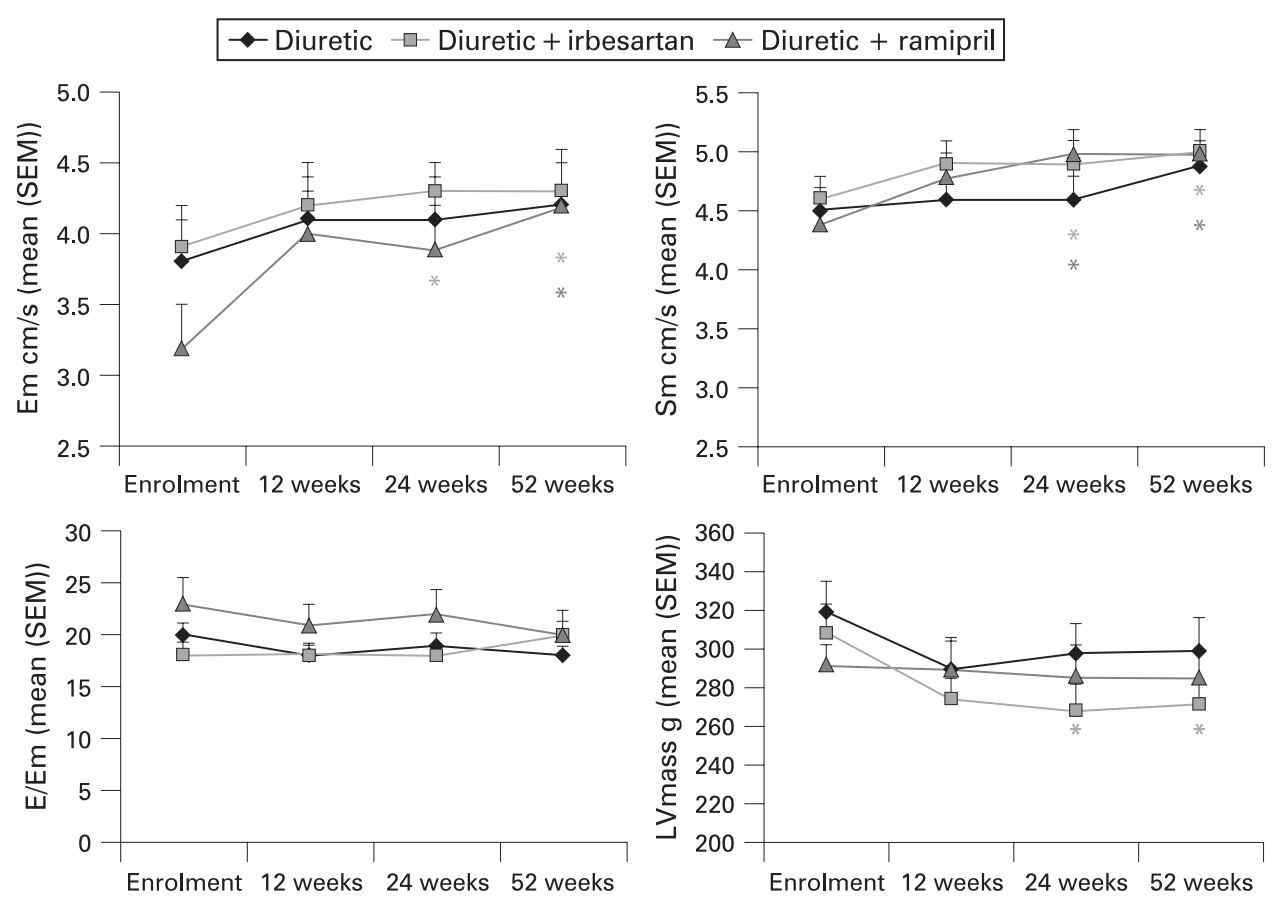


Table 3 Doppler echocardiographic results in DHF and normal control groups

\begin{tabular}{lrrc}
\hline & Normal & DHF & p Value \\
\hline Number & 38 & 151 & \\
Female/Male & $24 / 14$ & $93 / 58$ & \\
Age (years) & $72(7)$ & $74(7)$ & 0.11 \\
IVSd (cm) & $1.2(0.2)$ & $1.4(0.3)$ & 0.001 \\
LVEDD (cm) & $4.4(0.5)$ & $4.9(0.7)$ & 0.001 \\
LVESD (cm) & $2.9(0.5)$ & $3.4(0.7)$ & 0.068 \\
FS $(\%)$ & $36(6)$ & $32(8)$ & 0.005 \\
LVEF2d (\%) & $62(8)$ & $67(10)$ & 0.12 \\
LVmass (g) & $211(61)$ & $305(94)$ & $<0.001$ \\
LAD (cm) & $3.4(0.4)$ & $4.1(0.7)$ & $<0.001$ \\
E (m/s) & $0.67(0.2)$ & $0.65(0.2)$ & 0.52 \\
A (m/s) & $0.79(0.2)$ & $0.92(0.2)$ & $<0.001$ \\
E/A & $0.9(0.3)$ & $0.7(0.3)$ & $<0.001$ \\
DT (ms) & $200(63)$ & $259(77)$ & $<0.001$ \\
IVRT (ms) & $100(18)$ & $117(32)$ & $<0.001$ \\
Sm (cm/s) & $5.6(1.0)$ & $4.5(1.2)$ & $<0.001$ \\
Em (cm/s) & $5.7(1.5)$ & $3.7(1.6)$ & $<0.001$ \\
Am $(\mathrm{cm} / \mathrm{s})$ & $7.9(1.3)$ & $6.4(1.5)$ & $<0.001$ \\
E/Em & $12(3)$ & $20(9)$ & $<0.001$ \\
\hline
\end{tabular}

Results expressed as mean (SD).

\section{NT-pro-BNP}

The mean NT-pro-BNP level of all patients in the study was raised at baseline (595 (SD 904) pg/ml) although there was a wide scatter from $5-4748 \mathrm{pg} / \mathrm{ml}$ (table 2). The levels tended to fall in all treatment groups by 12 months although this reached statistical significance only in the irbesartan and ramipril groups.

\section{DISCUSSION}

In this study we found, first, that in HFNEF diuretics alone appeared to be effective in reducing symptoms and improving quality of life, and the addition of ramipril or irbesartan was not obviously more efficacious; second, that all therapies had only a slight effect on exercise capacity as assessed by the 6-minute walk test; and third, that ventricular annular or basal peak velocities are reduced in both systole and diastole and that these improve slightly after diuretics with ramipril or irbesartan.

Symptoms are an important part of the heart failure syndrome and quality of life is often severely impaired. Many patients with HFNEF are elderly and often frail, and for them therapy which improves symptoms or exercise capacity immediately may be more important than an uncertain possibility of a small reduction in mortality in the future. Relief of symptoms is, therefore, an important target of therapy but, being subjective, this is difficult to evaluate. A number of health-related quality of life instruments have evolved in order to assess the impact of disease, effect of treatment and other variables affecting people's lives. In this study we used a diseasespecific instrument - the Minnesota Living with Heart Failure (MLHF) questionnaire. ${ }^{15} \mathrm{~A}$ recent review has compared the MLHF with two other questionnaires and shown that the MLHF was more sensitive than the Chronic Heart Failure questionnaire and the generic abbreviated short form health survey ${ }^{21}$ In this study we used a Chinese version of the MLHF which has been validated in previous studies. ${ }^{16}$ It is likely, therefore, that the marked reduction in the MLHF score does truly represent an improvement in quality of life. Interestingly, we found that diuretics alone were almost equally as effective as diuretics combined with irbesartan or ramipril.
Table 4 Doppler echocardiographic results in the three groups

\begin{tabular}{|c|c|c|c|c|}
\hline & \multicolumn{4}{|l|}{ Diuretic group } \\
\hline & Baseline & 12 weeks & 24 weeks & 52 weeks \\
\hline IVSd (cm) & $1.39(0.04)$ & $1.37(0.05)$ & $1.38(0.05)$ & $1.34(0.04)^{*}$ \\
\hline LVEDD $(\mathrm{cm})$ & $4.90(0.11)$ & $4.83(0.11)$ & $4.78(0.12)$ & $4.81(0.10)$ \\
\hline LVESD (cm) & $3.28(0.12)$ & $3.34(0.13)$ & $3.28(0.12)$ & $3.30(0.11)$ \\
\hline LVEF (\%) & $0.69(0.02)$ & $0.66(0.02)$ & $0.67(0.02)$ & $0.67(0.02)$ \\
\hline LV mass $(\mathrm{g})$ & 309.85 (14.27) & $290.85(16.04)$ & $297.90(15.46)$ & $299.52(17.38)$ \\
\hline LAD (cm) & $3.85(0.08)$ & $3.76(0.11)$ & $3.74(0.10)$ & $3.74(0.10)$ \\
\hline $\mathrm{E}(\mathrm{m} / \mathrm{s})$ & $0.67(0.03)$ & $0.68(0.03)$ & $0.70(0.04)$ & $0.69(0.03)$ \\
\hline$A(\mathrm{~m} / \mathrm{s})$ & $0.91(0.03)$ & $0.89(0.03)$ & $0.90(0.03)$ & $0.94(0.03)$ \\
\hline $\mathrm{E} / \mathrm{A}$ & $0.74(0.05)$ & $0.77(0.05)$ & $0.75(0.04)$ & $0.73(0.04)$ \\
\hline IVRT (ms) & $117.93(6.00)$ & $121.49(5.62)$ & $114.08(4.02)$ & $113.73(3.44)$ \\
\hline $\mathrm{Sm}(\mathrm{cm} / \mathrm{s})$ & $4.57(0.22)$ & $4.55(0.20)$ & $4.64(0.21)$ & $4.89(0.21)^{*}$ \\
\hline $\mathrm{Em}(\mathrm{cm} / \mathrm{s})$ & $3.95(0.31)$ & $4.14(0.27)$ & $4.20(0.29)$ & $4.24(0.27)$ \\
\hline \multirow[t]{3}{*}{$\mathrm{E} / \mathrm{Em}$} & $24.83(5.64)$ & $20.40(3.03)$ & $27.26(8.74)$ & $19.61(2.06)$ \\
\hline & \multicolumn{4}{|c|}{ Diuretic + Irbesartan group } \\
\hline & Baseline & 12 weeks & 24 weeks & 52 weeks \\
\hline IVSd (cm) & $1.41(0.04)$ & $1.34(0.04)$ & $1.29(0.04)^{*}$ & $1.28(0.04)^{*}$ \\
\hline $\operatorname{LVEDD}(\mathrm{cm})$ & $4.94(0.12)$ & $4.84(0.08)$ & $4.82(0.08)$ & $4.81(0.10)$ \\
\hline LVESD (cm) & $3.41(0.10)$ & $3.27(0.08)$ & $3.33(0.09)$ & $3.29(0.08)$ \\
\hline LVEF (\%) & $0.66(0.01)$ & $0.68(0.02)$ & $0.66(0.01)$ & $0.67(0.01)$ \\
\hline LV mass $(\mathrm{g})$ & 310.25 (14.86) & $274.90(10.79)^{*}$ & $274.35(14.50)^{*}$ & ${ }^{*} 278.23(15.03)^{*}$ \\
\hline LAD (cm) & $4.12(0.11)$ & $3.88(0.11)^{*}$ & $3.97(0.11 \hat{)}$ & $4.01(0.15)^{*}$ \\
\hline$E(\mathrm{~m} / \mathrm{s})$ & $0.62(0.03)$ & $0.67(0.03)$ & $0.70(0.03)^{*}$ & $0.74(0.03)^{*}$ \\
\hline$A(\mathrm{~m} / \mathrm{s})$ & $0.93(0.03)$ & $0.92(0.03)$ & $0.93(0.04)$ & $0.96(0.03)$ \\
\hline$E / A$ & $0.64(0.03)$ & $0.71(0.05)$ & $0.80(0.1)^{*}$ & $0.72(0.03)^{*}$ \\
\hline IVRT (ms) & $113.06(3.84)$ & $113.20(3.19)$ & $115.66(3.30)$ & $109.21(3.42)$ \\
\hline $\mathrm{Sm}(\mathrm{cm} / \mathrm{s})$ & $4.54(0.17)$ & $4.84(0.16)$ & $4.88(0.17)^{*}$ & $4.92(0.16)^{* *}$ \\
\hline $\mathrm{Em}(\mathrm{cm} / \mathrm{s})$ & $3.79(0.25)$ & $4.06(0.25)$ & $4.20(0.23)^{*}$ & $4.16(0.25)^{*}$ \\
\hline \multirow[t]{3}{*}{$\mathrm{E} / \mathrm{Em}$} & $18.47(1.30)$ & $18.24(1.02)$ & $19.64(1.49)$ & $20.17(1.36)$ \\
\hline & \multicolumn{4}{|c|}{ Diuretic + Ramipril group } \\
\hline & Baseline & 12 weeks & 24 weeks & 52 weeks \\
\hline IVSd (cm) & $1.38(0.04)$ & $1.34(0.04)$ & $1.37(0.05)$ & $1.33(0.05)$ \\
\hline $\operatorname{LVEDD}(\mathrm{cm})$ & $4.84(0.08)$ & $4.87(0.08)$ & $4.73(0.10)$ & $4.80(0.10)$ \\
\hline LVESD (cm) & $3.36(0.06)$ & $3.37(0.07)$ & $3.22(0.09)$ & $3.28(0.08)$ \\
\hline LVEF (\%) & $0.65(0.01)$ & $0.67(0.01)$ & $0.68(0.01)$ & $0.67(0.01)$ \\
\hline LVmass (g) & 292.43 (1020) & 289.99 (13.62) & $285.97(15.56)$ & 285.68 (12.33) \\
\hline LAD $(\mathrm{cm})$ & $3.99(0.12)$ & $3.90(0.11)$ & $3.81(0.10 \hat{)}$ & $3.77(0.12)$ \\
\hline$E(\mathrm{~m} / \mathrm{s})$ & $0.66(0.03)$ & $0.70(0.04)$ & $0.71(0.04)$ & $0.69(0.03)$ \\
\hline$A(\mathrm{~m} / \mathrm{s})$ & $0.93(0.04)$ & $0.94(0.03)$ & $0.91(0.03)$ & $0.96(0.04)$ \\
\hline$E / A$ & $0.70(0.04)$ & $0.71(0.03)$ & $0.76(0.05)$ & $0.70(0.03)$ \\
\hline IVRT (ms) & $119.57(4.99)$ & 118.36 (3.12) & $110.82(3.85)^{*}$ & $110.45(3.97)$ \\
\hline $\mathrm{Sm}(\mathrm{cm} / \mathrm{s})$ & $4.46(0.24)$ & $4.76(0.22)$ & $5.02(0.22)^{*}$ & $4.94(0.2)^{*}$ \\
\hline $\mathrm{Em}(\mathrm{cm} / \mathrm{s})$ & $3.27(0.25)$ & $3.88(0.27)^{*}$ & $3.81(0.26)^{*}$ & $4.04(0.32)^{* * *}$ \\
\hline $\mathrm{E} / \mathrm{Em}$ & $24.01(2.99)$ & $20.11(1.73)$ & $20.98(2.03)$ & $19.07(2.07)^{*}$ \\
\hline
\end{tabular}

Results expressed as mean (SEM).

${ }^{* *} p<0.01 ;{ }^{*} p<0.05$ (significant differences between baseline and follow-up at 12, 24 or 52 weeks by paired $t$ tests with Bonferroni adjustment).

The symptomatic improvement was quite rapid and $70-90 \%$ of this had occurred by 12 weeks (table 2). There was a trend towards quicker progress with the addition of irbesartan or ramipril but there was no significant difference by 1 year. However, the observation that diuretics alone can produce a major impact on quality of life suggests that many of the symptoms in HFNEF are related to fluid overload and raised ventricular and atrial pressures. Maurer et al have found on the basis of LV pressure-volume analysis that HFNEF patients are a very heterogeneous group, that different pathophysiological mechanisms exist, and that in some the estimated end-diastolic pressure-volume relationship was shifted rightwards towards 
Table 5 Comparison between three groups at 52 weeks (percentile change from baseline)

\begin{tabular}{lrrrl}
\hline & \multicolumn{1}{l}{ Diuretic } & Diuretic + Irbesartan & Diuretic + Ramipril & p Value \\
\hline LVmass & $-11(-22$ to 10$)$ & $-8(-25$ to 11$)$ & $-7(-21$ to 18$)$ & 0.67 \\
Sm & $6.4(-6$ to 26$)$ & $12.5(0.2$ to 31.28$)$ & $5.5(-8.8$ to 29$)$ & 0.76 \\
Em & $4.4(-10$ to 49$)$ & $19(-7$ to 33$)$ & $27(-1$ to 63$)$ & 0.16 \\
E/Em & $-0.4(-18$ to 21$)$ & $13(-15$ to 31$)$ & $-5(-31$ to 17$)$ & 0.08 \\
\hline
\end{tabular}

Results expressed as median (interquartile range).

Comparison using Kruskal-Wallis test.

increased volumes consistent with a mild volume overload state rather than the typical DHF paradigm. ${ }^{22}$ A combination of cardiac and many extra cardiac factors such as renal function and venous tone, amongst others, may cause the rapid rise in LV filling pressures and pulmonary oedema.

Despite the significant improvement in quality of life scores in this study, the changes in exercise capacity were relatively modest. The 6-minute walk test is commonly used in heart failure clinical trials. Intuitively, walking on level ground would seem to be a more suitable test for elderly patients. However, the ability of the 6-minute walk test (MWT) to distinguish between effective and ineffective treatments has been questioned. In a systematic review of trials that have used the 6MWT, Olsson et $a^{24}$ concluded that the test has not yet been proven to be robust enough for the identification of effective pharmacological intervention. However, Olsson et al did find that the results of the 6MWT are usually concordant with changes in symptoms. ${ }^{24}$ In our study the directional change, if not the magnitude, in 6MWT was compatible with the symptoms score. However, in this group of elderly patients many other factors limit mobility and any improvement of cardiac function alone may not be sufficient to impact significantly on exercise capacity.

Ventricular long axis function is an important component of overall ventricular function. Recent studies using MRI have shown that longitudinal atrioventricular plane displacement accounts for $60 \%$ of stroke volume even in those with depressed systolic function due to dilated cardiomyopathy. ${ }^{14}$ Loss of longitudinal function may be compensated for by increased radial motion in the early stages. ${ }^{13}$ Previous studies have shown that both the peak systolic myocardial velocity $(\mathrm{Sm})$ and the peak early diastolic myocardial velocity $(\mathrm{Em})$ are reduced in HFNEF, despite a normal LVEF, challenging the concept that HFNEF/DHF is a distinct entity due to isolated diastolic dysfunction, ${ }^{4}$ and therefore in many patients systolic function cannot be considered to be truly normal in HFNEF/DHF. Not unexpectedly, given the fundamental role of mitral annular motion in ventricular function, both $\mathrm{Sm}$ and $\mathrm{Em}$ are powerful predictors of prognosis in a variety of cardiac conditions, including heart failure. ${ }^{12} 1825$ Furthermore, in a study of suspected heart failure, the echocardiographic parameter which best correlated with NT-proBNP was LV longitudinal peak systolic velocity. ${ }^{26}$ In an experimental model of heart failure Em declined early and before radial derived early diastolic velocities. ${ }^{27}$ This may be because motion of the ventricular base during early diastole is in effect reflecting both systolic and diastolic ventricular function. Em correlates well with tau, which is a measure of LV relaxation, but the mitral ring motion starts at the same time as mitral inflow, that is, when the pressure has already fallen. ${ }^{28}$ The early diastolic annular velocity (Em) is therefore primarily a measure of recoil or ventricular restoring forces, which depend to some extent on the nature of the previous systolic contraction, the degree of twist and untwist, incoordination and dyssynchrony.
How therapy with diuretics plus either irbesartan or ramipril improved long axis ventricular function without any appreciable change of LV ejection fraction cannot be exactly deduced from this study. There are a number of possible reasons including a reduction of LV mass, myocardial fibrosis, subendocardial ischaemia and afterload. It is known that arterial load affects both systolic and diastolic LV performance, prolonging contraction and relaxation (an effect seen early in the progression of systolic dysfunction), and shortening the diastolic filling period. ${ }^{29}$ Arterial compliance is also an independent predictor of diastolic dysfunction in patients with hypertensive heart disease. ${ }^{30}$ The effect of increased afterload or raised BP would be particularly troublesome for diastolic filling when heart rates are higher, such as with exercise and in those with atrial fibrillation. Thus, part of the improvement in peak myocardial systolic and diastolic velocities could be due to a reduction in arterial BP, which was observed in all treatment groups.

There was a trend for irbesartan and ramipril therapy in combination with diuretics to reduce LV mass, which became statistically significant only for irbesartan at 24 weeks, although by 1 year there was no difference between the three groups. Systolic blood pressure was lowered to a similar extent by all treatments, although diastolic blood pressure was reduced slightly more by irbesartan. In the Losartan intervention for End-point Reduction in Hypertension (LIFE) trial, losartan induced greater reduction in LV mass index from baseline than the $\beta$-blocker atenolol measured by echocardiography. ${ }^{31}$ ACE inhibitors also reduced LV mass in the Treatment Of Mild Hypertension study (TOMHS). ${ }^{32}$ In the VALIDD study antihypertensive therapy reduced blood pressure and improved diastolic relaxation velocity, but there was no significant difference between those receiving the angiotensin receptor blocker valsartan and those receiving a matching placebo. ${ }^{33}$ There have been no direct head-to-head comparisons of an angiotensin receptor antagonist with ACE inhibitors regarding their effect on LVH. However, LVH regression may be a clinically important end point, since regression of hypertensive $\mathrm{LVH}$ is associated with improved prognosis.

LV fibrosis is increased with LVH and hypertension ${ }^{34}$ and this is associated with reduced peak myocardial velocities. ${ }^{12}$ Shan et al found that both Sm and Em were inversely related to the percentage of interstitial fibrosis in endomyocardial biopsy specimens. ${ }^{35}$ ACE inhibitors and ARBs can block the fibrogenic action of angiotensin experimentally ${ }^{36}$ and have been shown to reduce fibrosis in patients with hypertension. ${ }^{37} 38$ Fibrosis and altered collagen in LVH may have a profound effect on overall myocardial architecture, and in particular ventricular twist and torsion, which is reflected in reduced longitudinal velocities, and this will impact directly on diastolic filling. Reducing fibrosis may, therefore, be an important therapeutic target in patients with LVH and heart failure or HFNEF. 


\section{Limitations}

This study has some limitations. Recruitment was slow as many potential patients were ineligible because of significant comorbidities, particularly renal dysfunction, valvular heart disease (which is common in this age group), anaemia, already treated with an ACE inhibitor or angiotensin receptor antagonist (usually because of previous myocardial infarction), frailty and dementia. These are typical for this age group. In addition, the strict entry criteria in this study, which required chest $x$-ray evidence of pulmonary oedema, were also a hurdle. Our experience does highlight the difficulty in performing clinical trials in this group of patients if the criteria for heart failure are strict. However, this study does have strengths: the study population is a representative sample with respect to age and aetiology; the diagnosis required $x$-ray confirmation of pulmonary oedema on admission, and other treatments that may have interfered, such as $\beta$-blockers and calcium antagonists, were not allowed; confirmation of the diagnosis comes from raised NT-proBNP levels in the majority despite earlier treatment with diuretics (to levels above those recommended in the recent revision of the European Society of Cardiology guidelines ${ }^{39}$ ); $\mathrm{E} / \mathrm{Em}$ ratios were increased, which is another diagnostic criterion in the new guidelines. ${ }^{39}$ Using colour tissue velocity imaging (which produces the mean velocity rather than peak as with pulsed tissue Doppler imaging) the ratio $\mathrm{E} / \mathrm{Em}$ is higher but a ratio $>15$ is still considered diagnostic. ${ }^{40}$ Using averaged velocity measurements from two or more sites is also recommended, as was done in this study.

In summary, this study is the first comparison of an ACE inhibitor and an angiotensin receptor blocker in patients with heart failure and normal ejection fraction. In addition, we have compared both of these drugs with diuretics alone. We have shown that diuretics alone can improve quality of life and symptoms in this group of patients and there was little further symptomatic benefit with the addition of irbesartan or ramipril. In addition, we have confirmed in a group of patients with HFNEF (DHF) that ventricular long axis function is reduced in both systole and diastole and that this was improved slightly by treatment with irbesartan or ramipril in combination with a diuretic.

Acknowledgements: This study was initially supported by a small grant from the manufacturers of Irbesartan, who also donated the irbesartan medication (SanofiSynthelabo). Design, conduct, retention of data, analysis and writing were all entirely independent and carried out by the authors only. Data were kept at the Chinese University of Hong Kong and are available for public scrutiny. None of the authors received any lecture, advisory board, or consultancy fees relating to this study from the sponsors. The study was registered at the Clinical Trials Unit at the Chinese University of Hong Kong (unique trial number CUHK CCT00035).

Competing interests: None.

\section{REFERENCES}

1. Hogg K, Swedberg K, McMurray J. Heart failure with preserved left ventricular systolic function: epidemiology, clinical characteristics, and prognosis. J Am Coll Cardiol 2004;43:317-27.

2. Sanderson JE. Review: Heart Failure with normal ejection fraction. Heart 2007;93:155-8.

3. Yusuf S, Pfeffer MA, Swedberg K, et al, for the CHARM Investigators and Committees. Effect of candesartan in patients with chronic heart failure and preserved left-ventricular ejection fraction: the CHARM-Preserved Trial. Lancet 2003;363:777-81.

4. Cleland JG, Tendera M, Adamus J, et al, PEP-CHF investigators. The perindopril in elderly people with chronic heart failure (PEP-CHF) study. Eur Heart J 2006;27:233845.

5. Yip G, Wang M, Zhang Y, et al. Left ventricular long axis function in diastolic heart failure is reduced in both diastole and systole: time for a redefinition? Heart 2002;87:121-5.

6. Petrie MC, Caruana L, Berry C, et al. "Diastolic heart failure" or heart failure caused by subtle left ventricular systolic dysfunction? Heart 2002;87:29-31.
7. Yu CM, Lin $\mathrm{H}$, Yang $\mathrm{H}$, et al. Progression of systolic abnormalities in patients with "isolated" diastolic heart failure and diastolic dysfunction. Circulation 2002;105:1195-201.

8. Nikitin NP, Witte KK, Clark AL, et al. Color tissue Doppler-derived long-axis left ventricular function in heart failure with preserved global systolic function. Am J Cardiol 2002;90:1174-7.

9. Bruch C, Gradaus R, Gunia S, et al. Doppler tissue analysis of mitral annular velocities: evidence for systolic abnormalities in patients with diastolic heart failure. J Am Soc Echocardiogr 2003;16:1031-6.

10. Vinereanu D, Nicolaides E, Tweddel AC, et al. "Pure" diastolic dysfunction is associated with long-axis systolic dysfunction. Implications for the diagnosis and classification of heart failure. Eur J Heart Fail 2005;7:820-8.

11. Garcia EH, Perna ER, Farias EF, et al. Reduced systolic performance by tissue Doppler in patients with preserved and abnormal ejection fraction: new insights in chronic heart failure. Int J Cardiol 2006;108:181-8.

12. Wang M, Yip GW, Wang AY, et al. Tissue Doppler imaging provides incremental prognostic value in patients with systemic hypertension and left ventricular hypertrophy. J Hypertens 2005;23:183-91.

13. Vinereanu D, Nicolaides E, Tweddel AC, et al. Subclinical left ventricular dysfunction in asymptomatic patients with Type II diabetes mellitus, related to serum lipids and glycated haemoglobin. Clin Sci (Lond) 2003;105:591-9.

14. Carlsson M, Ugander M, Mosen $\mathrm{H}$, et al. Atrioventricular plane displacement is the major contributor to left ventricular pumping in healthy adults, athletes, and patients with dilated cardiomyopathy. Am J Physiol Heart Circ Physiol 2007;292:H1452-9.

15. Rector TS, Kubo SH, Cohn JN. Patients' self-assessment of their congestive heart failure: content, reliability, and validity of a new measure, the Minnesota Living with Heart Failure Questionnaire. Heart Failure 1987;3:198-209.

16. Sanderson JE, Chan SKW, Yip G, et al. Beta-blockade in heart failure: a comparison of carvedilol with metoprolol. J Am Coll Cardiol 1999;34:1522-8.

17. Yusuf S, Ysuyuki R. Using exercise endpoints in heart failure trials: design considerations. Eur Heart J 1996;17:4-6.

18. Wang M, Yip GWK, Wang AYM, et al. Peak early diastolic mitral annulus velocity by tissue Doppler imaging adds independent and incremental prognostic value. J Am Coll Cardiol 2003:41:820-6.

19. Devereux RB, Alonso DR, Lutas EM, et al. Echocardiographic assessment of left ventricular hypertrophy: comparison to necropsy findings. Am J Cardiol 1986;57:450 8.

20. Nagueh SF, Middleton KJ, Kopelen HA, et al. Doppler tissue imaging: a noninvasive technique for evaluation of left ventricular relaxation and estimation of filling pressures. J Am Coll Cardiol 1997;30:1527-33.

21. Bennett SJ, Oldridge NB, Eckert GJ, et al. Comparison of quality of life measures in heart failure. Nurs Res 2003;52:207-16.

22. Maurer MS, Spevack D, Burkhoff D, et al. Diastolic dysfunction: can it be diagnosed by Doppler echocardiography? J Am Coll Cardiol 2004;44:1543-9.

23. Maurer MS, Kronzon I, Burkhoff D. Ventricular pump function in heart failure with normal ejection fraction: insights from pressure-volume measurements. Prog Cardiovasc Dis 2006;49:182-95.

24. Olsson LG, Swedberg K, Clark AL, et al. Six minute corridor walk test as an outcome measure for the assessment of treatment in randomized, blinded intervention trials of chronic heart failure: a systematic review. Eur Heart $J$ 2005;26:749-50.

25. Wang M, Yip GWK, Yu CM, et al. Independent and incremental prognostic value of early diastolic annular velocity in patients with impaired left ventricular systolic function. J Am Coll Cardiol 2005;45:272-7.

26. Vinereanu D, Lim P0, Frenneaux MP, et al. Reduced myocardial velocities of left ventricular long-axis contraction identify both systolic and diastolic heart failure-a comparison with brain natriuretic peptide. Eur J Heart Fail 2005; 7:512-9.

27. Hasegawa $\mathbf{H}$, Little WC, Ohno $\mathrm{M}$, et al. Diastolic mitral annular velocity during the development of heart failure. J Am Coll Cardiol 2003:41:1590-7.

28. Gibson DG, Francis DP. Clinical assessment of left ventricular diastolic function. Heart 2003;89:231-8.

29. Little WC. Enhanced load dependence of relaxation in heart failure. Circulation 1992;85:2326-8.

30. Mottram PM, Haluska BA, Leano R, et al. Relation of arterial stiffness to diastolic dysfunction in hypertensive heart disease. Heart 2005;91:1551-6.

31. Devereux RB, Dahlof B, Gerdts E, et al. Regression of hypertensive left ventricular hypertrophy by losartan compared with atenolol: the Losartan Intervention for Endpoint Reduction in Hypertension (LIFE) trial. Circulation 2004;110:1456-62.

32. Neaton JD, Grimm Jr RH, Prineas RJ, et al. Treatment of Mild Hypertension Study: final results. JAMA 1993;270:713-24.

33. Solomon SD, Janardhanan R, Verma A, et al. Effect of angiotensin receptor blockade and antihypertensive drugs on diastolic function in patients with hypertension and diastolic dysfunction: a randomized trial. Lancet 2007;369:2079-87

34. Weber KT, Brilla CG. Pathological hypertrophy and cardiac interstitium: fibrosis and renin-angiotensin-aldosterone system. Circulation 1991;83:1849-65.

35. Shan K, Bick RJ, Poindexter BJ, et al. Relation of tissue Doppler derived myocardial velocities to myocardial structure and beta-adrenergic receptor density in humans. J Am Coll Cardiol 2000;36:891-6. 
36. Weber KT. Targeting pathological remodeling. Concepts of cardioprotection and reparation. Circulation 2000;102:1342-5.

37. Brilla CG, Funck RC, Rupp H. Lisinopril-mediated regression of myocardial fibrosis in patients with hypertensive heart disease. Circulation 2000;102:1388-93.

38. Diez J, Querejeta R, Lopez B, et al. Losartan-dependent regression of myocardial fibrosis is associated with reduction of left ventricular chamber stiffness in hypertensive patients. Circulation 2002;105:2512-7.
39. Paulus WJ, Tschope C, Sanderson JE, et al. How to diagnose diastolic heart failure: a consensus statement on the diagnosis of heart failure with normal left ventricular ejection fraction by the Heart Failure and Echocardiography Associations of the European Society of Cardiology. Eur Heart J 2007:28:2539-50.

40. Dokainish H, Zoghbi WA, Lakkis NM, et al. Optimal non-invasive assessment of left ventricular filling pressures: a comparison of tissue Doppler echocardiography and Btype natriuretic peptide in patients with pulmonary artery catheters. Circulation 2004;109:2432-9.

\section{Images in cardiology}

\section{Failed endothelialisation of a percutaneous atrial septal defect closure device}

A 77-year-old woman with severe myxomatous mitral regurgitation underwent mitral valve replacement. Twenty-six months previously, she had undergone percutaneous closure of an atrial septal defect (ASD) with an Amplatzer device. At the time of surgery inspection of both sides of the septal occluder device revealed bare Nitinol wires suggesting failed endothelialisation (Panel A).

The use of percutaneous closure devices for the treatment of atrial septal defects is increasing. However, our current knowledge regarding the long-term safety and efficacy of these devices is limited. This case illustrates that endothelialisation of such devices may be significantly delayed or even absent following implantation, raising the possibility of late thromboembolic complications. Presently there is no consensus of opinion regarding antiplatelet therapy following device implantation. ${ }^{1}$ In the United Kingdom, general guidelines issued in 2004 regarding the percutaneous implantation of ASD closure devices estimated the incidence of device-associated thrombus formation at $0.4-3 \%$, based on data from large non-randomised studies. ${ }^{2}$ However, these studies differed in the type of device implanted and the antiplatelet agent regimens employed. We routinely perform transoesophageal echocardiography 6 months postprocedure prior to stopping dual antiplatelet therapy, which is continued thereafter only if there has been a neurological event.

Non-endothelialised devices present potent substrates for thrombus formation and the potential for cerebrovascular accidents. This case illustrates a previously unrecognised and potentially serious problem, highlighting the unresolved issue of the appropriate type and duration of antiplatelet therapy, and the need for strategies to accelerate endothelial coverage of such occluder devices.

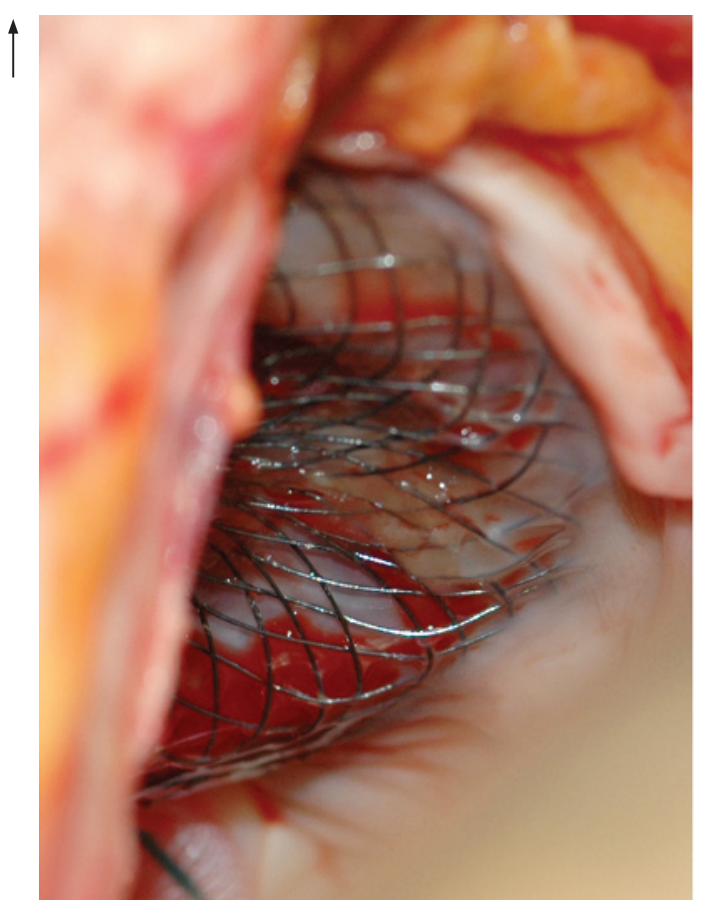

Panel A. Intraoperative image of the atrial septal defect closure device, viewed from the left atrium, showing the bare Nitinol mesh.

\section{Z Astroulakis, A El-Gamel, J M Hill}

jonathan.hill@kcl.ac.uk

Competing interests: None.

Heart 2008:94:580. doi:10.1136/hrt.2007.135251

\section{REFERENCES}

1. Franke A, Kühl HP. The role of antiplatelet agents in the management of patients receiving intracardiac closure devices. Curr Pharm Des 2006;12:1287-91.

2. Interventional procedure consultation document-endovascular closure of atrial septal defect. National Institute for Clinical Excellence. Interventional Procedures Programme, March 2004 